\title{
Perspectives of stem cells use in alzheimer's disease treatment
}

\begin{abstract}
The article contains analysis of perspectives of cell technologies use in the therapy of patients with Alzheimer's disease (AD). Currently there are no effective ways to diagnose this disease at early stage. Late diagnostics of $\mathrm{AD}$ and weak understanding of its mechanisms complicate clinical tactics leaving in fact palliative (symptomatic) treatment as the only available option. Authors propose a combination of the traditional methods of treatment with newly developed cell therapy technologies. Stem cells (SC) implantation is the basis for recovery of functional activity of neural networks in the regions affected by neurodegeneration. Based upon conducted experimental studies and analysis of scientific literature, the authors recommend to use the technique of the intranasal perineural application of autologous mesenchymal stem cells (MSC) for the treatment of patients with Alzheimer's disease. Following the administration into the submucosa of nasal cavity, SC migrate along the olfactory nerve to the brain promoting a reparative effect, particularly attributed to the excretion of neurotrophic factors (brain-derived neurotrophic factor (BDNF), nerve growth factor (NGF), insulin growth factor-1 (IGF-1), and vascular endothelial growth factor (VEGF)).
\end{abstract}

Volume 8 Issue 3 - 2018

\author{
Vladimir Kulchitsky, 'Alexandra Zamaro,' \\ Valeria Navitskaya, ${ }^{2}$ Yuri Shanko, ${ }^{2}$ Svetlana \\ Pashkevich,' Yulya Stukach,' Stanislav \\ Koulchitsky ${ }^{3}$ \\ 'Institute of Physiology, National Academy of Sciences of \\ Belarus, Belarus \\ ${ }^{2}$ Republican Centre of Neurology and Neurosurgery of Ministry \\ of Health, Belarus \\ ${ }^{3}$ Liege University, Belgium
}

Correspondence: Vladimir Kulchitsky, Institute of Physiology, National Academy of Sciences of Belarus, Belarus, Email vladi@fizio.bas-net.by

Received: May 07, 2018 | Published: June 19, 2018

Keywords: alzheimer's disease, cell technology, intranasal implantation, stem cell, therapy

\section{Abbreviations: AD, alzheimer's disease; BDNF, brain- derived neurotrophic factor; IGF-1, insulin growth factor-1; MSC, mesenchymal stem cells; NGF, nerve growth factor; SC, stem cells; VEGF, vascular endothelial growth factor \\ Introduction}

Serious cognitive dysfunctions in $\mathrm{AD}$ patients result from disruptions of neural circuitry due to failure in coordinated development of neural processes provoked by pathological events at the intra-and extracellular brain levels..$^{1-3}$ One of the key factors playing a role in this pathology is a defect in amyloid precursor protein excretion. ${ }^{1-3}$ Excessive accumulation of amyloid beta in the brain is accompanied by the formation of plaques around the nerve cells. Noticeably, pathological processes develop not only in the intracellular matrix but also inside the neurons: it was shown that the content of microtubule-associated tau protein extremely increases in $\mathrm{AD}$ patients causing interruption of normal neural networks activity. ${ }^{1,2}$ Based on these data, scientists and physicians are trying nano-and immunotherapy, anti-inflammatory and neuroprotective drugs along with removal of amyloids and tau protein excess off the brain, but no relief in brain's activity is observed. ${ }^{1,2}$

One possible reason of the inefficacy of the current therapies is the absence of effective diagnostics at early stages. As a consequence, the therapeutic procedures start too late, when the pathological process is irreversible, and significant amount of central neurons has been already lost. ${ }^{2}$ This explanation of course does not downgrade the frustration of physicians and patients. What can we suggest regarding the issue? No doubt, it is advisable to go on searching for early markers of neurodegenerative processes and investigation of their underlying mechanisms. At the same time it would be reasonable to implement some new therapies promoting the replenishment of neurons. ${ }^{1-9}$
We are not the first who pay attention to $\mathrm{SC}$ as a promising way of anti-AD therapy. PubMed search for "stem cell Alzheimer's disease" on May-06-2018 returned 1839 scientific articles, most of them published within the recent years. However, we have to note that almost all the authors try to use systemic (mainly intravascular), ${ }^{2-4,9}$ and intracerebral ${ }^{8}$ ways of $\mathrm{SC}$ administration in their experiments. Unfortunately, these methods of SC administration have low efficacy when applied to the patients with various brain diseases. In the cases of systemic injection, SC fail to pass the blood-brain barrier, which reduces their migration to the injured brain regions. Therefore, we suggest local administration of MSC into submucosa of nasal cavity allowing their natural perineural migration to the target regions. . $^{6,10,11}$

In this context, a wide range of different methods of cell therapy should be tested: embryonic SC, induced pluripotent SC, MSC-all these cells have positive potential in the treatment of patients with AD. ${ }^{2,7,9,12}$ Positive effect of SC's neurotrophic function is well known: they release cytokines, growth factors and other signaling molecules (BDNF, NGF, IGF-1, VEGF) in damaged brain regions. ${ }^{1,3,5,6}$ A number of authors suggests that these trophic factors released by SC in the impaired areas can stimulate the reparative mechanisms. ${ }^{1,3,5,6,9}$ Some promising results have been received in the studies using animal $\mathrm{AD}$ models; these experiments demonstrated the ability of SC to differentiate into various types of neuronal and glial cells. . $, 9,12^{2}$

As was mentioned above, the search of biological markers allowing early $\mathrm{AD}$ diagnosis is also of critical importance. For example, AD is associated with the diffuse interruption of acetylcholinesterase synthesis in occipital and frontal brain regions. A very similar phenomenon was detected after the destruction of olfactory bulbs cells in experiments on Wistar rats. Noticeably, this was accompanied by the lowering of visual perception, decrease of olfactory sensitivity to irritating smells, and impairment of memory performance in 
Morris water maze tests. ${ }^{13}$ Interestingly, the pool of SC located at the area of olfactory bulbs is involved in post-stroke recovery of brain functions..$^{10,11}$ Altogether, this gives an idea that such parameters as visual perception, olfactory sensitivity and memory, although far not specific, might be used to detect the early degenerative changes in the $\mathrm{CNS}$ of the potential AD patients.

Nevertheless, several issues in the field of diagnostics and treatment of $\mathrm{AD}$ are still waiting to be verified and resolved in order for SC therapy to become viable for patients. Viability of this technique for therapy of $\mathrm{AD}$ requires thorough experimental verification in order to conclude on real effectiveness of intranasal perineural migration of MSC in clinical practice.

\section{Acknowledgements}

This pooled analysis was funded by OOO "Synergy", and by grant B18A3-025 of BRFFI.

\section{Conflict of interest}

All listed authors concur with the submission of the manuscript; all authors have approved the final version. The authors have no financial or personal conflicts of interest.

\section{Refernces}

1. Amemori T, Jendelova P, Ruzicka J, etal.Alzheimer's disease: mechanism and approach to cell therapy. Int J Mol Sci. 2015;16(11):26417-26451.

2. Cacabelos R. Have there been improvements in Alzheimer's disease drug discovery over the past 5 years? Expert Opine Drug Discov. 2018.13(6):523-538.

3. Chen WW, Blurton-Jones M. Concise review: can stem cells be used to treat or model Alzheimer's disease? Stem Cells. 2012;30(12):26122618 .
4. Honmou O, Houkin $\mathrm{K}$, Matsunaga $\mathrm{T}$, et al. Intravenous administration of auto serum-expanded autologous mesenchymal stem cells in stroke. Brain. 2011;134(pt 6):1790-1807.

5. Kwak KA, Lee SP, Yang JY, et al. Current Perspectives regarding stem cell-based therapy for Alzheimer's disease. Stem Cells Int. 2018;6392986:14.

6. Lee JH, Oh IH, Lim HK. Stem cell therapy: a prospective treatment for Alzheimer's disease. Psychiatry Investig. 2016;13(6):583-589.

7. Ming L, Kequan G, Susumu I. Stem cell treatment for Alzheimer's disease. Int J Mol Sci. 2014;15(10):19226-19238.

8. Kulchitsky V, Zamaro A, Shanko Y, et al. Positive and negative aspects of cell technologies in cerebral diseases. J Neurol Stroke. 2008;8(2):8788.

9. Tong LM, Fong H, Huang Y. Stem cell therapy for Alzheimer's disease and related disorders: current status and future perspectives. Exp Mol Med. 2015;47:e151.

10. Zamaro A, Stukach Yu, Panakhova E, et al. The visual and olfactory signs of neurodegenerative diseases. IEEE Intern Symposium. «Video and Audio Signal Processing in the Context of Neurotechnologies». Petersburg, Russia; 2016. p. 44-47.

11. Stukach YP, Shanko YG, Kulchitsky VA. Experimental substantiation of stem cells delivery to the brain through cerebral nerves endings. Biological motility. 2016. p. 232-235.

12. Stukach Y. Stem cells migration to the brain through cranial nerves endings. Euro Biotech J. 2017;1(1):91-92.

13. Ma T, Gong K, Ao Q, et al. Intracerebral transplantation of adiposederived mesenchymal stem cells alternatively activates microglia and ameliorates neuropathological deficits in Alzheimer's disease mice. Cell Transplant. 2013;22(Suppl 1):S113-126. 